\title{
Synchrotron emission from secondary leptons in microquasar jets
}

\section{P. Bordas*}

Departament d'Astronomia i Meteorologia, Universitat de Barcelona, Martí i Franquès 1, 08028, Barcelona, Spain

E-mail: pbordas@am.ub.es

\section{J. M. Paredes ${ }^{\dagger}$}

Departament d'Astronomia i Meteorologia, Universitat de Barcelona, Martí i Franquès 1, 08028, Barcelona, Spain

E-mail: jmparedes@ub.edu

\section{Bosch-Ramon}

Max Planck Institut für Kernphysik, Saupfercheckweg 1, Heidelberg 69117, Germany

E-mail: vbosch@mpi-hd.mpg.de

\section{Orellana $\S$}

Instituto Argentino de Radioastronomía, CC5, (1894) Villa Elisa, Buenos Aires, Argentina. E-mail: morellanadirma.iar.unlp.edu.ar

The decay of charged pions produced in proton-proton collisions between stellar wind ions and relativistic protons in microquasar (MQ) jets can generate secondary leptons that emit through detectable synchrotron radio emission. Energy losses due to synchrotron and inverse Compton (IC) processes, and adiabatic expansion are taken into account. We present a model to compute the synchrotron emission using particle energy distributions that are consistently derived. We present the Spectral Energy Distributions (SEDs) while exploring the space parameter in our model. Our conclusions show a significant though hardly dominant contribution of secondary leptonic emission to the total radio emission. On the other hand, observational consequences obtained can be used to test some still unknown processes occurring in these objects as well as the nature of the matter outflowing in their jets.

VI Microquasar Workshop: Microquasars and Beyond

September 18-22 2006

Società del Casino, Como, Italy

\footnotetext{
* Speaker.

${ }^{\dagger}$ P.B and J.M.P acknowledge support by DGI of the Spanish Ministerio de Educación y Ciencia (MEC) under grant AYA2004-07171-C02-01, as well as partial support by the European Regional Development Fund (ERDF/FEDER).

$¥$ V.B-R. thanks the Max-Planck-Institut für Kernphysik for its support and kind hospitality.

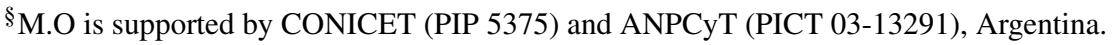




\section{Introduction}

X-ray binary systems (XRBs) are composed by a compact object (either a stellar mass black hole or a neutron star) and a normal (non degenerated) star that supplies matter to the compact object through the formation of an accretion disk. Some $\sim 260$ XRBs are known up to now [1], probably corresponding to an underlying population of some tens of thousands of compact objects in the whole Galaxy. Some radio emitting X-ray binaries (REXBs) have been observed to display jets like those seen in quasars and active galactic nuclei but up to distances $\sim 10^{-6}$ times smaller. This analogy is the reason for calling them microquasars (MQs) [2]. Attention on these objects has grown since the proposal of Paredes et al. (2000) [3] of MQs as counterparts of some of the unidentified gamma-ray sources of the EGRET catalog [4] and hence pointing them as plausible high energy emitters. A strong confirmation of this association has come from the detections of the MQs LS 5039 and LS I +61 303 at Tev energies using respectively the ground-based Cherenkov telescopes HESS [5] and MAGIC [6], giving support to a number of previous studies centered on the mechanisms operating in these sources in order to explain the gamma ray domain (see, e.g., [7] and [8]). A jet origin of the emission from MQs has been suggested from the observation of syncrothron emission of relativistic electrons/positrons extending from the radio all the way into the $\mathrm{X}$-ray regime. In the framework of secondary leptons generated through hadronic interactions, the present work focuses on the modelisation of the secondary leptonic synchrotron emission in order to constrain the characterization of MQ hadronic jets. An outline of the model is given in the next, followed by the results showing the SEDs and lightcurves under different parameter assumptions and the conclusions that can be extracted from them.

\section{Model description}

Our physical scenario is composed by a high-mass early-type star who feeds the accretion transfer of mass onto a compact object while developing an accretion disk. A relativistic $e^{ \pm}-p$ plasma is ejected in a direction taken to be perpendicular to the plane defined by the disk. Jet energetics is assumed to be dominated by accretion. The jet will also contain a magnetic field $B_{j e t}$ associated with the plasma. We assume that the matter kinetic luminosity is higher than the magnetic luminosity (or total magnetic energy crossing the jet section per time unit) in the jet regions we are concerned with. In this scenario we deal with radiative processes that take place in the jet and can produce significant emission in the radio spectral band. We focus on the leptonic component only although the contribution of protons could still be significant from the radiative point of view. Emission produced by the primary hadrons can be found elsewhere (see, e.g., [8] and [9]).

\subsection{Secondary generation and evolution}

Secondary leptons are generated in $p$ - $p$ interactions of hot protons in the jet with the companion star wind that extents out isotropically producing charged and neutral pions $\left(\pi^{ \pm}, \pi^{0}\right)$ through the reaction channel $p+p \rightarrow p+p+\xi_{\pi^{0}} \pi^{0}+\xi_{\pi^{ \pm}}\left(\pi^{+}+\pi^{-}\right)$, where $\xi_{\pi^{ \pm}} \sim 2\left(E_{p} / G e V\right)^{1 / 4}$ is the charged pion multiplicity. The relativistic injection proton spectrum is a power law $N_{p}\left(E_{p}\right)=$ $K_{p} E_{p}^{-\alpha}$ where $E_{p}^{\min } \leq E_{p} \leq E_{p}^{\max }$, and the constant $K_{p}$ can be found normalizing to the total power 
Table 1: Adopted parameter values.

\begin{tabular}{lcc}
\hline parameter & Symbol & Value \\
\hline & & \\
Black hole mass & $M_{b h}$ & $3 M_{\odot}$ \\
Injection point & $z_{0}$ & $50 R_{g}$ \\
Initial radius & $R_{0}$ & $5 R_{g}$ \\
Radius of the companion star & $R_{\star}$ & $15 R_{\odot}$ \\
Orbital radius & $a$ & $3 R_{\star}$ \\
Luninosity companion star & $L_{\star}$ & $1.6 \times 10^{39} \mathrm{erg} / \mathrm{s}$ \\
Mass loss rate & $\dot{M}_{\star}$ & $3 \cdot 10^{-6} M_{\odot} \mathrm{yr}{ }^{-1}$ \\
Jet's Lorentz factor & $\Gamma$ & 1.02 \\
Jet kinetic luminosity & $Q_{j}$ & $10^{36} \mathrm{erg} / \mathrm{s}$ \\
Proton kinetic Luminosity & $Q_{p}$ & $10^{35} \mathrm{erg} / \mathrm{s}$ \\
Minimum proton energy & $E_{p}^{\min }$ & $2 \mathrm{GeV}$ \\
Maximum proton energy & $E_{p}^{\max }$ & $100 \mathrm{TeV}$ \\
Leptonic spectral index & $P$ & 2.2 \\
Wind-jet penetration factor & $f_{p}$ & 0.1 \\
Magnetic field at $z_{0}$ & $B_{0}$ & $10^{2}, 10^{3}, 10^{4}$ \\
& & \\
\hline
\end{tabular}

that goes to protons, $Q_{p}$. The proton flux will be given by $J_{p}\left(E_{p}\right)=(c / 4 \pi) K_{p}\left(z_{0} / z\right)^{2} E_{p}^{-\alpha}$. We assume that a fraction of $1 / 10$ of the matter that crosses the jet region penetrates into it. When charged and neutral pions are created, the first will decay to muons and subsequently to electrons and positrons, while the second will decay to high energy photons. For an injection proton spectrum as the one given above, the pion spectrum (in the jet reference frame) will be approximately a power law and the electron/positron distribution will also follow (approximately) a power law [10] with a differential pair injection rate given by $J_{e}\left(E_{e}\right)=K_{e} E_{e}^{-P}$. Here we use a value $P=2.2$.

This injected electron-positron population suffers subsequent radiative cooling due to synchrotron and inverse Compton losses as well as to adiabatic expansion. Fresh electrons and positrons that have been injected at a certain point into the jet suffer these losses modifying in this way the spectral distribution of particles at each height $z$. Moreover, different evolution stages sum at each height since injection of fresh particles occurs all along the jet and therefore a mix of multiple (differently evolved) population of electron/positron distribution arises. To compute it in a consistent way, the continuity equation $N_{e^{ \pm}}\left(E_{e^{ \pm}}, z\right) d E_{e^{ \pm}}=N_{0, e^{ \pm}}\left(E_{0, e^{ \pm}}, z_{0}\right) d E_{0, e^{ \pm}}$is used where $N_{0, e^{ \pm}}\left(E_{0, e^{ \pm}}, z_{0}\right)=K_{0, e^{ \pm}} E_{0, e^{ \pm}}^{-p}$ is the initial energy spectrum for the injected particle density. Solving the equations for the evolution of the particle energy along the jet axis, one can find the spectral distribution at each slice, compute the differential luminosity at each height and finally integrate to find the total luminosity. 

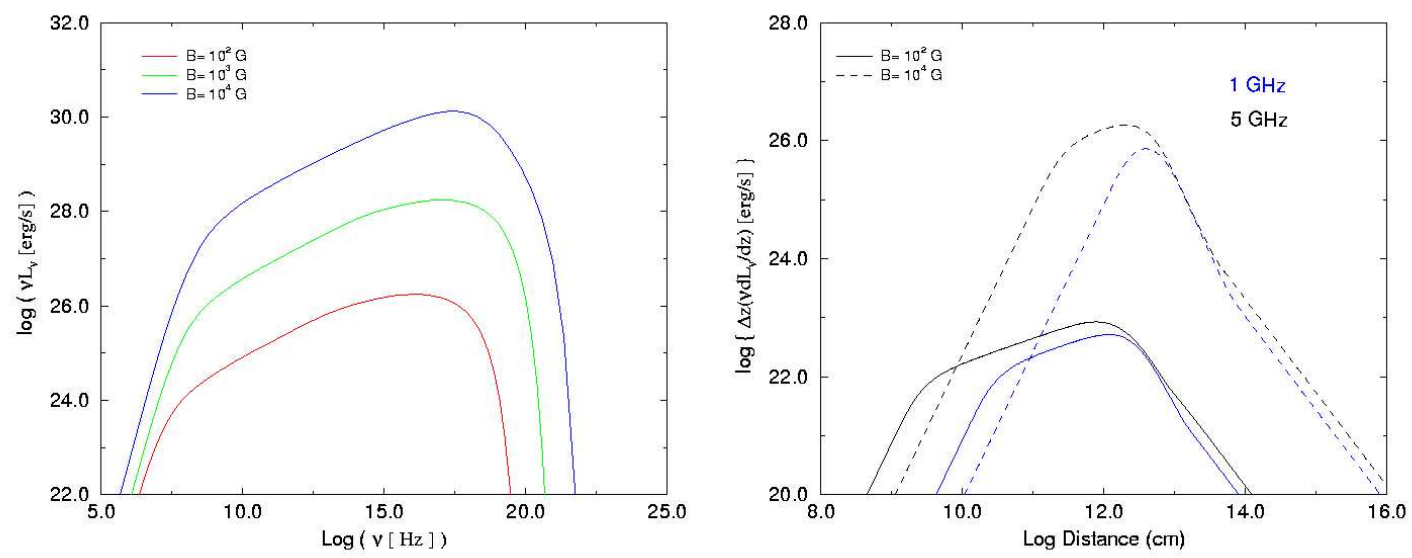

Figure 1: Left The strength of the magnetic field $B$ is treated as a free parameter in our model. Synchrotron losses will be enhanced when taking higher values for $B$ but, since injection occurs all along the jet inside the bynary system (until a distance roughly given by $z \simeq R_{o r b} \sim 10^{13} \mathrm{~cm}$ ), the enhancement in the final emission will depend on the synchrotron/IC loss balance. Here we show the SEDs for three different magnetic fields; values indicated are taken at the base of the jet. Right Light curves of emission at 1 and $5 \mathrm{GHz}$ bands at growing distances along the jet for two different magnetic fields $B$ wich values are given at the base of the jet at $z_{o}=50 R_{g}$. Not only the overall emission but also the peak of the lightcurves moves to higher distances when increasing $B$, as well as the difference between the locations of the peaks for the two frequencies

\subsection{Secondary emission}

Secondary electrons and positrons will radiate through synchrotron process since we assume the presence of a magnetic field wich is tangled to the plasma and oriented randomly in direction. The resulting emission will be isotropic in the jet reference frame; to compute the expected luminosity, we have used the formulae presented in [11], where expressions for the specific emission and absorption coefficients, $\varepsilon_{v}$ and $k_{v}$ respectively, can be found. Then, the specific differential luminosity at a certain heigh $z$ in the jet can be expressed as $d L_{v}(z) / d z=2 \pi R(z) \frac{\varepsilon_{v}}{k_{v}}\left(1-e^{-l_{j} k_{v}}\right)$ where $l_{j}$ is the typical size of the synchrotron emitting plasma region and $R(z)$ is the jet radius at each heigh $z$. Now, integrating over the entire jet lenght and taking into account the Doppler boosting factor $\delta=\left[\Gamma\left(1-\beta \cos \theta_{o b s}\right)\right]^{-1}$ we can finally find the SEDs in the observer reference frame.

\section{Model results and conclusions}

SEDs for different magnetic field values and emission along the jet at 1 and $5 \mathrm{GHz}$ are obtained. We have taken into account in a consistent way particle injection mechanisms and cooling due to radiation processes and adiabatic expansion. The luminosities obtained are slightly lower than in the models based on primary lepton injection, and must be considered complementary to them. However, we note that within our model there are no requirements of acceleration processes along the jet to obtain the milliarcsecond scale extended emission. The fact of studying alternative models were particles are directly injected until a certain height along the jet can constrain the amount of acceleration required and contribute to the understanding of the physical mechanisms 
that can lead to such processes. Signatures at different distances along the jet have the potential to be an important clue for determining the matter content of jets. In particular, high resolution observations at 1 and $5 \mathrm{GHz}$ could determine if leptons are present at heights $10^{12-13} \mathrm{~cm}$ at the edge of the binary system typical region where wind matter from the companion is still significant. If electrons/positrons still show high energies due to a recent injection from hadronic interactions at these parts of the jet, it could be a signature of secondary generation for which it would not be required to invoke additional acceleration processes.

\section{References}

[1] Liu, Q.Z., van Paradijs, J., van den Heuvel, E.P.J.: Catalogue of high-mass X-ray binaries in the Galaxy (4th edition) A\&A, 455, 1165-1168 (2006)

[2] Mirabel, I.F., Rodríguez, L.F.: Sources of Relativistic Jets in the Galaxy. ARA\&A, 37, 409-443 (1999)

[3] Paredes, J.M., Martí, J., Ribó, M., Massi, M.: Discovery of a High-Energy Gamma-Ray-Emitting Persistent Microquasar. Science 288, 2340-2342 (2000)

[4] Hartman, R.C., Bertsch, D.L., Bloom, S.D. et al.: Third EGRET catalog (3EG), ApJS, 123, 79 (1999)

[5] Aharonian, F., Akhperjanian, A.G., Aye, K.M. et al.: Discovery of Very High Energy Gamma Rays Associated with an X-ray Binary. Science 309, 746-749 (2005)

[6] Albert, J., Aliu, E., Anderhub, H., Antoranz, P. et al.: Variable Very-High-Energy Gamma-Ray Emission from the Microquasar LS I +61 303 Science 312, 1771-1773 (2006)

[7] Bosch-Ramon, V., Paredes,J.M., High-energy $\gamma$-ray Emission from Microquasars: LS 5039 and LS I +61 303 CHJAA 5, 133-138 (2005)

[8] Romero, Gustavo E., Christiansen, Hugo R., Orellana, M.: Hadronic High-Energy Gamma-Ray Emission from the Microquasar LS I +61 303 ApJ 632, Issue 2, 1093-1098 (2005)

[9] Romero, G.E., Torres, D.F., Kaufman Bernadó, M., Mirabel, I.F.: Hadronic gamma-ray emission from windy microquasars, A\&A, 410, L1-L4 (2003)

[10] Ginzburg, V.L., Syrovatskii, S.I.: The Secondary Electron Component of Cosmic Rays and the Spectrum of General Galactic Radio Emission. Soviet Astronomy, 8, 342, (1964)

[11] Pacholczyk, A.G.: Nonthermal processes in galactic and extragalactic sources; San Francisco, Freeman (1970) 